\title{
Effect of nano-encapsulated (-) epigallocatechin gallate on triglyceride accumulation in 3T3-L1 adipocytes
}

\author{
Priyanka Bapat, Shu Wang \\ From Metabolism, diet and disease \\ Washington, DC, USA. 29-31 May 2012
}

\section{Background}

Obesity is considered the major risk factor for type 2 diabetes mellitus (T2DM), cardio-vascular diseases like hypertension and atherosclerosis [1]. Catechins are the major source of flavonols and they are comprised of various types of catechins such as epigallo catechin 3 gallate (EGCG), epigallocatechin (EGC), epicatechin gallate (ECG) [2]. Numbers of in vivo studies have reported weight loss after drinking green tea for several months. To support these in vivo findings, many in vitro studies demonstrated effect of pure EGCG which is an important biologically active green tea catechin in reducing triglyceride accumulation in adipocytes [3]. However, these antiadipogenic properties of EGCG are greatly dependent on bio-availability which is very low in animals as well as in humans. One study showed that, peak plasma concentrations in humans were $1.3 \mu \mathrm{M}$ for EGCG after a single dose of $1.5 \mathrm{mmol}$ through oral administration [4]. Nanoencapsulated EGCG (Nano-EGCG) may help to increase bioavailability and to exert maximum anti-adipogenic effects.

\section{Materials and methods}

We synthesized Nano-EGCG using sonication method. Pure EGCG (95\%) was bought from SIGMA Company. Nano-EGCG was dissolved in phosphate buffered saline (1XPBS) and was stored at 4 degree Celsius. 3T3-L1 Preadipocytes were obtained from ATCC. Cells were cultured using a standard protocol. Post-confluence cells were differentiated using a standard chemical cocktail mixture. Further cells were maintained in maturation medium and treated with native EGCG and Nano-EGCG at $10 \mu \mathrm{M}$ for
48 hours. Cellular triglyceride levels were measured using an enzymatic method.

\section{Results}

Nano-EGCG significantly $(\mathrm{p}<0.05)$ reduced triglyceride accumulation in mature adipocytes as compared to $1 \mathrm{X}$ PBS, native EGCG and its void counterpart. EGCG didn't significantly reduce the cellular triglyceride accumulation as compared to $1 \mathrm{XPBS}$.

\section{Conclusion}

Data suggests that $10 \mathrm{uM}$ of Nano-EGCG significantly reduced triglyceride accumulation as compared to $1 \mathrm{X}$ PBS, void counterpart and native EGCG.

\section{Published: 1 June 2012}

\section{References}

1. Serrano Rios M: Relationship between obesity and the increased risk of major complications in non-insulin-dependent diabetes mellitus. Eur $J$ Clin Invest 1998, 28(Suppl 2):14-17, discussion 17-18.

2. Sano M, Tabata M, Suzuki M, Degawa M, Miyase T, Maeda-Yamamoto M: Simultaneous determination of twelve tea catechins by highperformance liquid chromatography with electrochemical detection. Analyst 2001, 126:816-820.

3. Lee MS, Kim CT, Kim Y: Green tea (-)-epigallocatechin-3-gallate reduces body weight with regulation of multiple genes expression in adipose tissue of diet-induced obese mice. Ann Nutr Metab 2009, 54:151-157.

4. Cabrera C, Artacho R, Gimenez R: Beneficial effects of green tea - a review. J Am Coll Nutr 2006, 25:79-99.

doi:10.1186/1753-6561-6-S3-P55

Cite this article as: Bapat and Wang: Effect of nano-encapsulated (-) epigallocatechin gallate on triglyceride accumulation in 3T3-L1 adipocytes. BMC Proceedings 2012 6(Suppl 3):P55. 